\title{
Transplantation of human amniotic mesenchymal stem cells in the treatment of focal cerebral ischemia
}

\author{
FANG LI ${ }^{1,2}$, ZONG-NING MIAO ${ }^{3}$, YUN-YUN XU ${ }^{4}$, SHI-YING ZHENG $^{5}$, \\ MING-DE QIN ${ }^{1}$, YAN-ZHENG GU ${ }^{1}$ and XUE-GUANG ZHANG ${ }^{1}$

\begin{abstract}
${ }^{1}$ Institute of Medical Biotechnology, Soochow University, Jiangsu Province Key Laboratory of Stem Cells;
${ }^{2}$ Department of Human Anatomy, Histology and Embryology, School of Biology and Basic Medical Sciences, Soochow University, Suzhou 215007; ${ }^{3}$ Stem Cell Research Lab of Wuxi, No. 3 People's Hospital, Wuxi 214041;

The First Affiliated Hospital of Soochow University, Suzhou 215006, P.R. China
\end{abstract} \\ ${ }^{4}$ Children's Hospital Affiliated to Suzhou University, Institute of Pediatrics; ${ }^{5}$ Department of Cardio-Thoracic Surgery,
}

Received February 6, 2012; Accepted June 15, 2012

DOI: $10.3892 / \mathrm{mmr} .2012 .968$

\begin{abstract}
Cerebrovascular injury is one of the three major causes of death and is the leading cause of adult disability. Despite the increasing progress in emergency treatment and early rehabilitation in patients with cerebrovascular injury, treatment options for neurological dysfunction that presents at a later stage are lacking. This study examined the potential of human amniotic mesenchymal stem cell (hAMSC) transplantation in the repair of neurological deficits in an experimental focal cerebral ischemia model. Following the isolation of hAMSCs, growth characteristics and surface antigen expression were observed. Butylated hydroxyanisole (BHA) was used to induce the cultured cells into neuron-like cells, which were identified by immunocytochemistry. The suture model was used to induce focal cerebral ischemia in rats, which were subsequently randomly divided into experimental and control groups for treatment with BrdU-labeled hAMSCs or PBS, respectively. Neurological deficits were assessed following transplantation using the neurological severity score, beam balance test and elevated body swing test. Eight weeks later, rat brain tissue was analyzed with $\mathrm{H} \& \mathrm{E}$ staining and $\mathrm{BrdU}$ immunohistochemistry, and the survival and spatial distribution of the transplanted hAMSCs were determined. The hAMSCs proliferated in vitro, and it was found that neuronspecific enolase (NSE) was expressed in neurons, whereas glial
\end{abstract}

Correspondence to: Dr Xue-Guang Zhang, Institute of Medical Biotechnology, Soochow University, Jiangsu Province Key Laboratory of Stem Cells, No. 1 Shixin Street, Canglang, Suzhou 215007, P.R. China

E-mail: xueguangzh@yahoo.com.cn

Dr Shi-Ying Zheng, Department of Cardio-Thoracic Surgery, The First Affiliated Hospital of Soochow University, Suzhou 215006, P.R. China

E-mail: syzheng88@sina.com

Key words: placenta mesenchymal, isolation and culture, focal cerebral ischemia, transplantation fibrillary acidic protein (GFAP) was expressed in astrocytes. The focal ischemia model caused varying degrees of left limb hemiplegia accompanied by right sided Horner's Syndrome. When examined 1,3, 6 and 8 weeks later, significant recovery in neurological behavior was detected in the rats treated with hAMSC transplantation compared with the control $(\mathrm{P}<0.01)$. BrdU-labeled hAMSCs were concentrated near the graft site and surrounding areas, in certain cases migrating towards the ischemic lesion. Local gliosis and lymphocytic infiltration were not detected. hAMSCs exhibit great potential for proliferation and are induced to differentiate into NSE-expressing neuron-like cells following treatment with BHA. Moreover, hAMSC transplantation may improve neurological symptoms following focal cerebral ischemia.

\section{Introduction}

Cerebrovascular injury is one of the three major causes of death and is the leading cause of adult disability. The annual incidence rate in China is 130-300 million, with 60-100 million deaths and $75 \%$ of survivors suffering disabilities of various degrees. Despite the increasing progress in emergency treatment and early rehabilitation in patients with cerebrovascular injury, treatment options for neurological dysfunction that presents at a later stage are lacking.

Regenerative medicine and stem cell research have progressed significantly in the 21 st century, offering novel routes for the treatment of neurological disorders. Mesenchymal stem cells (MSCs), unlike hematopoietic stem cells, are present in bone marrow. Bone mesenchymal stem cells (BMSCs) have become a progressive research field in modern biology and medicine. MSCs are derived from the mesoderm early in development and may be exploited as an ideal source of seed cells, which exhibit the potential to be induced into osteogenic, chondrogenic and adipogenic cells, or even tendon and adipose tissues (1-4). MSCs are easy to obtain, culture and expand in vitro, and are readily induced into designated tissues. Currently, BMSCs are widely used. However, MSCs are present in extremely low amounts in bone marrow, accounting for $0.01-0.001 \%$ of the bone marrow- 
derived cells (5). Increasing evidence indicates that MSCs with osteogenic potential may be isolated from a diverse range of tissues, including adipose (6) and perinatal tissues, such as umbilical cord $(7)$, placenta $(8,9)$, umbilical cord blood $(10,11)$ and amniotic fluid $(12,13)$, or even fetal blood, bone marrow and liver (14-17).

Placenta, a temporary organ, is important for maintaining maternal and fetal oxygen and nutrients during embryonic development. The full-term placenta comprises amnion and chorion, and our previous findings (18) indicate that MSCs may be obtained and expanded from the amnion (amniotic MSCs; AMSCs) and chorion (chorionic MSCs; CMSCs) of placenta (placental MSCs; PMSCs) in vitro; their biological characteristics remain well maintained, similar to those of BMSCs. In addition, a cell bank of PMSCs may be set up in advance for clinical trials, suggesting that PMSCs have a wide application prospect (18).

In this study, we aimed to establish a stable and reliable method for the isolation and amplification of human amniotic mesenchymal stem cells (hAMSCs) in vitro. Following induction into neural cells, the hAMSCs were transplanted into the ischemic tissue of rats subjected to focal cerebral ischemia by middle cerebral artery occlusion (MCAO). The survival, migration and differentiation of the implanted cells and the recovery of neurological function were assessed in rats 1-8 weeks later to examine the potential therapeutic benefit of the hAMSC-derived neuron-like cell transplantation in the treatment of focal cerebral ischemia.

\section{Materials and methods}

Cell culture. hAMSCs were obtained from normal post-partum placenta. The amnion and villus layer were bluntly separated and repeatedly washed with D-Hank's solution, including double resistant (100 U/ml penicillin and $100 \mu \mathrm{g} / \mathrm{ml}$ streptomycin). After rinsing, the amnion was cut into several 1x 1-mm sections with ophthalmic scissors and digested at $37^{\circ} \mathrm{C}$ in a water bath for $\sim 30 \mathrm{~min}$ with the action of $2.5 \mathrm{~g} / 1$ trypsin (Gibco-BRL, Carlsbad, CA, USA). The digestion of the amnion was terminated with Dulbecco's modified Eagle's medium (DMEM) containing 5\% calf serum and filtered through a 200 mesh cell sieve. The filtered amnion products were digested again in a $37^{\circ} \mathrm{C}$ water bath for $\sim 0.5 \mathrm{~h}$ with the addition of $1.0 \mathrm{~g} / \mathrm{l}$ collagenase II (Sigma-Aldrich, St. Louis, MO, USA). Subsequent termination and filtrations were performed as described above. Finally, the harvested cell suspensions were centrifuged at $1,000 \mathrm{rpm}$ for $5 \mathrm{~min}$ and the cell pellet was resuspended in lowglucose DMEM (L-DMEM; Hyclone Laboratories, Inc., South Logan, UT, USA) supplemented with $10 \%$ fetal bovine serum (FBS; Hyclone) and 1\% penicillin-streptomycin (Invitrogen Life Technologies, Carlsbad, CA, USA). The cells were then plated in $25-\mathrm{cm}^{2}$ culture flasks at a density of $1 \times 10^{6}$ cells $/ \mathrm{ml}$ and incubated at $37^{\circ} \mathrm{C}$ with $5 \%$ carbon dioxide. The medium was changed every 2 days. When the established adherent cell colonies reached $70 \%$ confluence, they were detached with $2.5 \mathrm{~g} / \mathrm{l}$ trypsin and replated at a ratio of $1: 2$ in $25-\mathrm{cm}^{2}$ flasks.

Differentiation of hAMSCs. Second or third generation hAMSCs were plated onto 6-well plates. When $60 \%$ confluence was achieved, the harvested cells were washed with phosphate- buffered saline (PBS). To induce neural differentiation, the hAMSCs were incubated with serum-free medium containing DMSO (2\%) and butylated hydroxyanisole (BHA) $(100 \mu \mathrm{M})$. The media were changed every 3 days and culturing was continued for 14-21 days. The neural induced cells were then confirmed by neuron-specific enolase (NSE) and glial fibrillary acidic protein (GFAP) immunofluorescence staining.

Immunofluorescence. Immunofluorescence was performed on hAMSCs cultured for $24 \mathrm{~h}$. The cells were grown to $60 \%$ confluence on 6-well plates, washed with PBS three times, fixed in $4 \%$ paraformaldehyde for $30 \mathrm{~min}$, washed as previously described, permeabilized in $0.3 \%$ Triton X-100 for 20 min and then rinsed with PBS three times. The cells were then blocked with goat serum for $20 \mathrm{~min}$, incubated with the appropriate primary antibody in PBS for $2 \mathrm{~h}$ at $37^{\circ} \mathrm{C}$, washed with PBS three times, incubated with secondary antibodies in PBS for $30 \mathrm{~min}$ at $37^{\circ} \mathrm{C}$ (in the dark) and then viewed under a fluorescence microscope. The following primary antibodies were used: rabbit anti-human NSE (1:500) and rabbit anti-human GFAP (1:500), both from Boster Biological Technology, Ltd). The secondary antibody for immunofluorescence was goat anti-rabbit IgG (1:500; Sigma).

BrdU labeling and preparation of cell suspension for transplantation. Third generation cells from the AMSCs were collected and plated in $25-\mathrm{cm}^{2}$ culture flasks and 6-well plates at a density of $1 \times 10^{5}$ cells $/ \mathrm{ml}$. The cell pellet was resuspended in L-DMEM supplemented with $10 \% \mathrm{FBS}$, penicillin-streptomycin $(100 \mu \mathrm{g} / \mathrm{ml}), 5 \mathrm{ng} / \mathrm{ml} \mathrm{bFGF}$ and $10 \mu \mathrm{g} / \mathrm{ml} \mathrm{BrdU}$, and then incubated at $37^{\circ} \mathrm{C}$ with $5 \%$ carbon dioxide for $48 \mathrm{~h}$. BrdUlabeled AMSCs were centrifuged at $1,000 \mathrm{rpm}$ for $10 \mathrm{~min}$ and the cell pellet was resuspended in PBS at $1 \times 10^{6}$ cells $/ \mu 1$. Finally, $5-\mu 1$ cell suspensions were used for cell transplantation.

Animal model. Healthy male Wistar rats, aged 3-4 months and weighing 250-300 g, were obtained from the Schistosomiasis Prevention and Control Center of Jiangsu Province, China. Briefly, the rats were placed in a supine position on an operating table following the intraperitoneal injection of $10 \%$ chloral hydrate $(4 \mathrm{ml} / \mathrm{kg})$ anesthetic. A blunt dissection of the sternocleidomastoid was made through the middle line neck incision, and the carotid artery (CCA) was isolated and then separated into the right external carotid artery (ECA), internal carotid artery (ICA) and the wing jaw artery. A slipknot was left under the ECA and the wing jaw artery, after threading deeply into all the arteries. The CCA was clamped, a small incision was made in the proximal sidewall of the ECA and a nylon suture filament $(0.24 \mathrm{~mm})$ was inserted and advanced to a depth of $\sim 18.5 \pm 0.5 \mathrm{~mm}$ away from the CCA bifurcation. The suture was removed following a 2-h right MCAO, the ECA was ligated and the skin was sutured.

Animal grouping. Out of 45 rats subjected to focal cerebral ischemia, 13 died and 8 did not exhibit paralysis of the limbs. The remaining 24 rats were randomly divided into two groups ( $n=12$ per group).

hAMSC transplantation. Two weeks after MCAO, the rats were placed in a stereotactic apparatus and the bregma was exposed through a median head scalp incision. Coordinates 
Table I. Neurological severity scores.

\begin{tabular}{cc}
\hline Grading & Score \\
(normal, 0; \\
maximum, 5)
\end{tabular}

Normal walk

0

Flexion of forelimb (raising the rat by the tail)

Circling toward the paretic side (walking)

Falling down to the paretic side (walking)

No spontaneous walking,

decreased consciousness

Ischemia-related deaths

1

2

3

4

5

Neurological function in the rats was assessed using the neurological severity scores (NSS) test. The higher the NSS score, the more severe the ischemia.

Table II. Beam balance test.

\begin{tabular}{cc}
\hline Grading & Score \\
$($ normal, $0 ;$ \\
maximum, 6)
\end{tabular}

Balances with steady posture

0

Grasps side of beam

1

Hugs the beam and one limb

falls down from the beam

Hugs the beam and two limbs fall down

3

from the beam, or spins on beam $(>60 \mathrm{sec})$

Attempts to balance on the beam,

but falls off (>40 sec)

Attempts to balance on the beam,

but falls off ( $>20 \mathrm{sec})$

Falls off: no attempt to balance or

hang on to the beam (>20 sec)

Neurological function in the rats was assessed using the beam balance test (BBT). The higher the BBT score, the more severe the ischemia.

were marked to enable targeting of the striatum (1 $\mathrm{mm}$ anterior to the skull, left margin 2.5 $\mathrm{mm}$; depth 4.5-5.5 $\mathrm{mm}$ ), and a $5 \mu \mathrm{l}$ BrdU-labeled AMSC suspension or PBS control was then injected into the striatum with a Hamilton syringe for $10 \mathrm{~min}$.

Neurological behavior evaluation. Neurological deficit evaluations were carried out prior to the transplantation and 1, 3, 6 and 8 weeks after MCAO using the neurological severity score (NSS; Table I), beam balance test (BBT; Table II) and elevated body swing test (EBST). For the EBST, observers were instructed to record data only when the rat head moved $>10^{\circ}$ from the vertical axis within $30 \mathrm{sec}$ of the rat being raised by the tail. This procedure was followed by $1 \mathrm{~min}$ of rest, and the test was then repeated 20 times. For the three tests, recordings were taken on every last day of weeks 1, 3, 6 and 8, and all rats were tested three times at different time-points for each test, and the average result was determined.
Triphenyltetrazolium chloride (TTC) staining. TTC staining was used to show the ischemic area of the brain tissue following $2 \mathrm{~h}$ of perfusion. A coronal cut was then made in the brain tissue, $2 \mathrm{~mm}$ behind the optic chiasm, the latter part of the brain was immersed in $1 \%$ TTC (Sigma-Aldrich) in PBS at $37^{\circ} \mathrm{C}$ for $30 \mathrm{~min}$ and then into $10 \%$ neutralized formalin overnight.

Preparation of paraffin and frozen sections. Eight weeks after MCAO, the rats were anesthetized intraperitoneally with $400 \mathrm{mg} / \mathrm{kg}$ chloral hydrate, perfused transcardially with $4 \%$ paraformaldehyde in PBS, and their brains were quickly extracted. An $\sim 2 \mathrm{~cm}$ ischemic area of brain tissue, including the lateral ventricles and basal ganglia, striatum and hippocampus, was excised and post-fixed in $4 \%$ paraformaldehyde. Coronal brain slices $(5-\mu \mathrm{m})$ were then consecutively sampled using paraffin sections or frozen sections.

Perl's Prussian Blue stain for hemosiderin. Sections were transferred to distilled water with xylene and ethanol, placed into the working solution (an equal parts mixture of ferrocyanide and hydrochloric acid) for $15 \mathrm{~min}$, rinsed with distilled water and then with tap water. Sections were then stained with neutral red for $1 \mathrm{~min}$, rinsed well with tap water, dehydrated with ethanol and finally cleared with xylene. Out of 400 slices, every 20 th slice was stained using this method to confirm the needle placement and injected sites.

Statistical analysis. Data were presented as the mean \pm standard deviation. Comparisons of neurological scores were carried out by ANOVA (F test, q test), using SPSS 10.0. The paired t-test was used for the cell count. In the analysis, a value of $\mathrm{P}<0.05$ indicated a statistically significant result.

\section{Results}

AMSC culture and cell phenotype. Adherent cells were observed $4 \mathrm{~h}$ after the cells were plated, and clone-like growth was observed $48 \mathrm{~h}$ later. The morphology of these cells was similar to that of BMSCs: spindle-shaped with fibroblast-like colonies adhering to the plastic surface. Flow analysis (Fig. 1) showed that the AMSCs expressed the typical MSC markers (CD73, CD105 and CD90), but were negative for hematopoietic markers (CD34 and CD45), the monocytic marker (CD14) and HLA-DR. A large number of BrdU-positive cells were observed using fluorescence microscopy, suggesting successful transplantation of the AMSCs.

Neural induction of AMSCs. Morphological changes, including condensed cell bodies with outgrowth in a few sites, were detected in some of the cells $2 \mathrm{~h}$ after incubation (Fig. 2A), with more cells showing these neural cell-like changes $1 \mathrm{~h}$ later (Fig. 2B). In addition to the morphological changes, differentiated cells expressed NSE, a marker for neural progenitor cells, and GFAP, a marker for astrocytes (Fig. 2C and D, respectively).

Neurological function score. The rats were tested for neurological function at different time-points using the NSS, BBT and EBST tests (Fig. 3A-C). In each test, the neurological behaviors were markedly improved, and there was a 

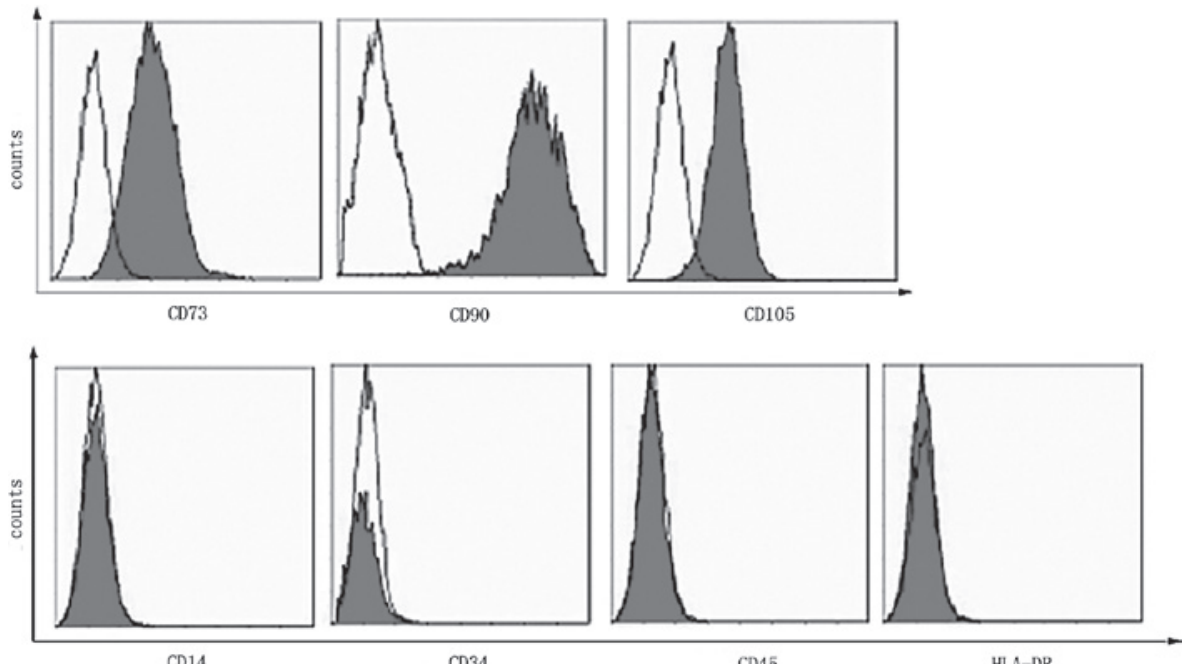

Figure 1. Surface expression of cultured AMSCs. Dark grey histograms showed positive cells, whereas black histograms showed IgG isotype control staining. AMSCs, amniotic mesenchymal stem cells.
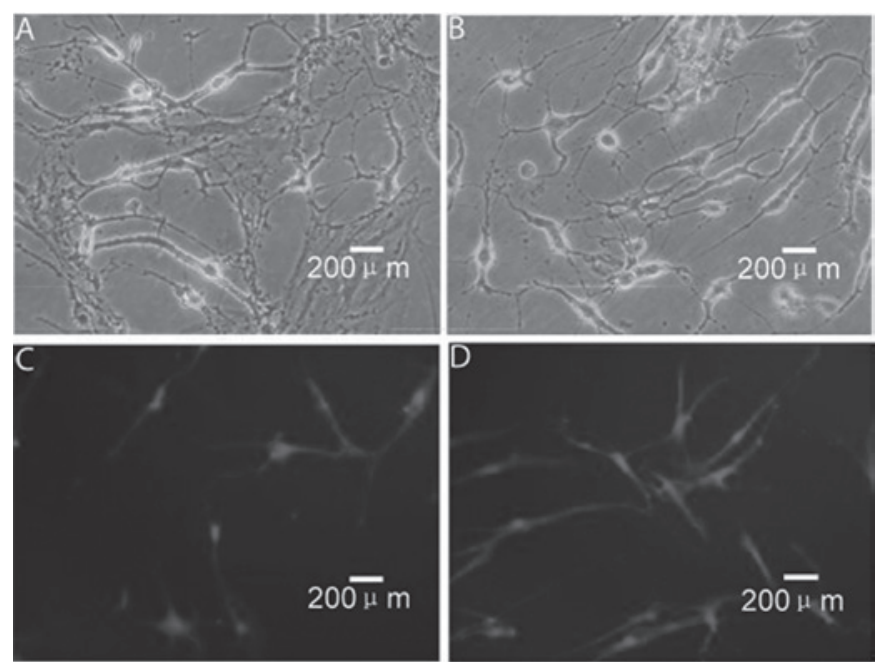

Figure 2. Neural differentiation of AMSCs. Morphological changes were observed by phase contrast microscopy after (A) $8 \mathrm{~h}$ and (B) $24 \mathrm{~h}$ of incubation. Immunocytochemical staining of (C) NSE and (D) GFAP showed that the differentiated cells with typical neural cell morphologies were positive (scale bar, $200 \mu \mathrm{m}$ ). AMSCs, amniotic mesenchymal stem cells; NSE, neuron-specific enolase; GFAP, glial fibrillary acidic protein.

significant difference between the AMSC-transplanted and the PBS-injected groups.

TTC and H\&E staining. TTC staining is a standard for the measurement of infarct size and has previously been used for assessment of infarct size resulting from apoptosis and necrosis (6). Normal brain tissue was shown in gray, while the ischemic area was white in TTC staining (Fig. 4D). The ischemic region was observed in the dorsolateral striatum and was lateral to the ischemic hemisphere, which is consistent with the blood supply area of the middle cerebral artery (Fig. 4A). In the ischemic hemisphere, $\mathrm{H} \& \mathrm{E}$ staining indicated a large necrotic area in the 8th week after transplantation. Within this region, there was a significant loss of neurons, with only a few remaining astrocytes, and a marked interstitial edema. The surviving neurons had varying degrees of morphological
A

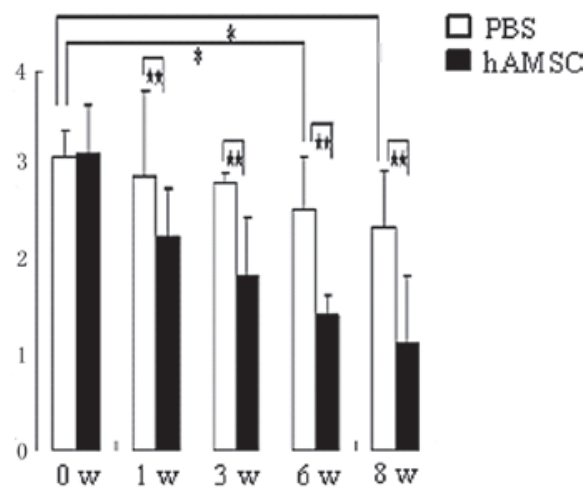

B

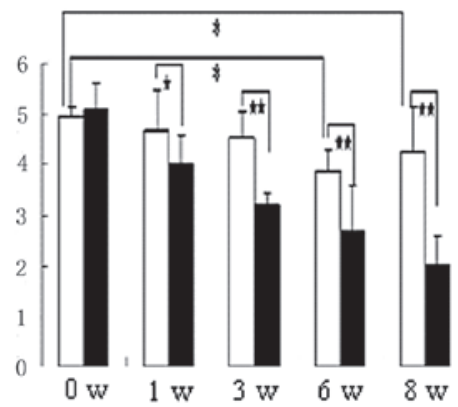

C

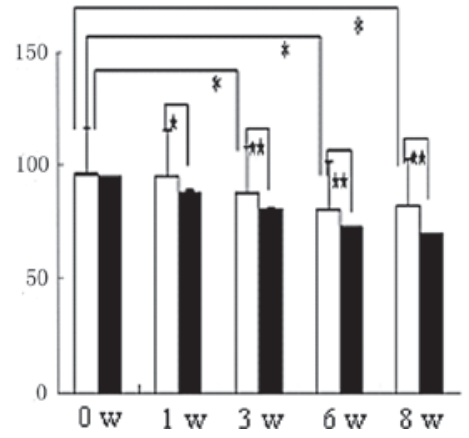

Figure 3. Results of (A) NSS, (B) BBT and (C) EBST showed that the neurological behaviors of the rats treated by hAMSC transplantation were significantly improved compared with the control group. hAMSC, human amniotic mesenchymal stem cells; NSS, neurological severity score; BBT, balance beam test; EBST, elevated body swing test. 


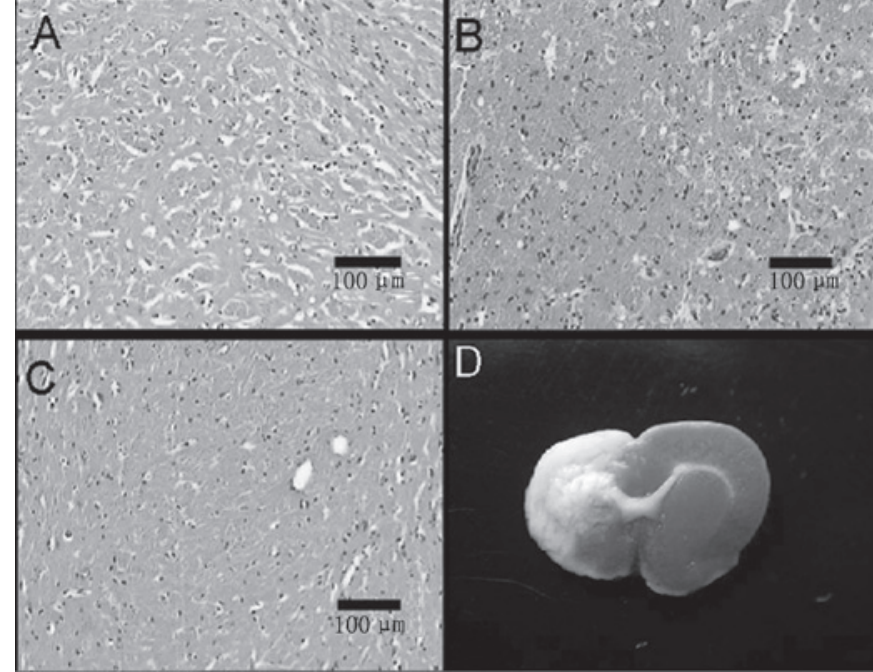

Figure 4. Histology of the MCAO area in the rats on the 8th week after transplantation. (A) H\&E staining showed the normal brain tissue of the rats (B) The ischemic area of the brain tissue from the control group (PBS-injected group). (C) The ischemic area of the brain tissue from the experimental group (AMSC-transplanted group). (D) TTC staining showed the ischemic area of the brain tissue (scale bar, $100 \mu \mathrm{m}$, in A, B and C). MCAO, focal cerebral ischemia; AMSC, amniotic mesenchymal stem cell.

changes, with the most significant changes occurring in pyramidal cells, which showed a shrunken cell body, retracted processes and loss of Nissl bodies. Furthermore, the chromatin became cloudy and the nuclear membrane decreased in size (Fig. 4B). Fewer degenerated cells were observed in the AMSC-transplanted area (Fig. 4C).

Determination of injection sites, needle passage and the BrdUlabeled transplanted AMSCs. The injection sites and needle tracks were identified in each of the 24 rats using specific hemosiderin staining (Fig. 5A) and showed that 17 rats were injected into the striatum and the other 7 rats in the cerebral cortex. Small amounts of lymphocytic infiltration and glial cell proliferation were observed around the needle tracks (Fig. 5B and C). In the experimental group, BrdU-positive AMSCs near the ischemic lesion were found to be distributed around the needle passages (Fig. 5D), with some cells residing at a distance of $2 \mathrm{~mm}$ away. No BrdU-positive cells were observed around the needle passages in the control group.

\section{Discussion}

Cell replacement therapy has become a developing and promising approach for the treatment of central nervous system injury and disease. In this study, we used the focal cerebral ischemia model in rats and implanted hAMSCs in the ischemic hemisphere using stereotaxic targeting to the striatum or cortex. We observed that cell survival and differentiation of the hPMSCs in the cerebral ischemic rat brain was associated with recovery of neurological function. We found that PMSCs implanted into ischemic tissue in rats resulted in improved neurological function and balance beam test performances relative to the control group. Similarly, histological staining showed PMSC survival within the ischemic region.

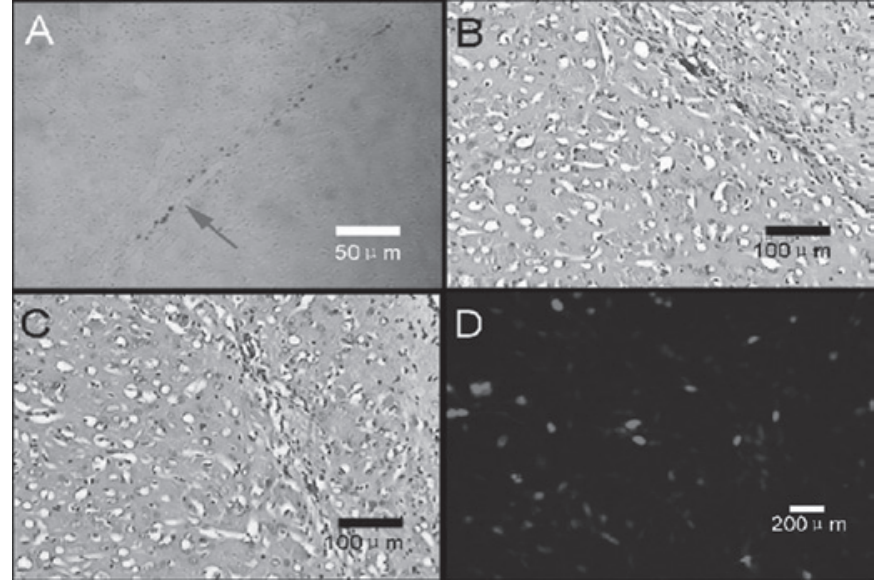

Figure 5. On the 8th week after transplantation, the injected sites and needle passage were shown by hemosiderin staining (the specific site was indicated by the arrow; scale bar, $50 \mu \mathrm{m}$ ) (A). More lymphocytic infiltration and glial cell proliferation were found around the needle passage for the PBS-injected group (B) than in the AMSCs-transplanted group (C) (H\&E staining; scale bar, $100 \mu \mathrm{m}$ ). (D) Under the fluorescence microscope, BrdU-positive AMSCs were found distributed around the needle passages (scale bar, $200 \mu \mathrm{m}$ ). PBS, phosphate-buffered saline; AMSCs, amniotic mesenchymal stem cells.

Silva et al analyzed the gene expression of MSCs and found that MSCs, not only code the genes of mesenchymal tissue, but also the genes of endothelial and epithelial tissues (19). These results provide a theoretical basis for the potential differentiation of MSCs. MSCs may be used to replace a variety of cells due to their inherent plasticity of cross-system and even crossgerm layer differentiation. Deng et al showed that the bone marrow MSCs of rats spontaneously express neural-specific proteins (20), such as NSE, $\beta$-III tubulin, NFM and S100- $\beta$. In this study, the expression of NSE and GFAP was detected following the induction of the hAMSCs by BHA. Furthermore, placental amnion develop from embryonic ectoderm, thus we speculated that the amniotic MSCs are more readily induced to differentiate into astrocytes and neuronal cells than are MSCs derived from other sources. Therefore, hAMSCs have broad application prospects in the treatment of nervous system damage and repair cell research.

When determining the best time-points for the transplantation of hAMSCs following ischemic injury, it is important to consider the release of toxic neurotransmitters and oxygen-free radicals at the early stage of transplantation, and the effect of scar formation on the growth and differentiation of the transplanted cells at chronic infarction. For example, Li et al found that when cells were transplanted 1 or 7 days after acute stroke, nerve toxins, free radicals and pro-inflammatory mediators led to the further development of ischemic injury and affected the transplanted cells which underwent apoptotic cell death in the ischemic penumbra (21). In addition, inflammation activates microglia and inhibits the growth and survival rate of endogenous neural cells. Fukunaga et al considered the best treatment window for BMSC transplantation to be at least 1 month after the patient experienced a stroke (22). In the present study, we transplanted cells 2 weeks after stroke and found that hAMSCs are dense within the ischemic lesion, suggesting that they migrate and/or proliferate within the injured tissue. Furthermore, we found that 8 weeks after cell transplantation, neurological function was improved compared with the control group. 
In this study, we showed that the transplantation of hAMSCs markedly improves neurological recovery following MCAO through stereotaxic injection. Additionally, that the recovery was likely to be associated with the secretion function of the implanted MSCs, as it has been reported that the ratio of cell survival and differentiation reaches approximately $80 \%$ in vitro (23) and only $3-10 \%$ in vivo (24). However, the mechanism underlying recovery is unclear and should be investigated. However, the approach we have described in this study offers a promising new route for the treatment of neurological disorders, including ischemic stroke.

\section{Acknowledgements}

This study was supported by grants from the Major State Basic Research Development Program of China (973 Program: 2007CB512402), the National Natural Science Foundation of China (nos. 30930085 and 31000654). The authors especially thank Dr Shan Jiang and Qun Xue for the valuable suggestions and critical review of this manuscript.

\section{References}

1. Jiang Y, Jahagirdar BN, Reinhardt RL, et al: Pluripotency of mesenchymal stem cells derived from adult marrow. Nature 418 : 41-49, 2002.

2. Kadiyala S, Young RG, Thiede MA, et al: Culture expanded canine mesenchymal stem cells possess osteochondrogenic potential in vivo and in vitro. Cell Transplant 6: 125-134, 1997.

3. Reyes M, Lund T, Lenvik T, et al: Purification and ex vivo expansion of postnatal human marrow mesodermal progenitor cells. Blood 98: 2615-2625, 2001.

4. Väänänen HK: Mesenchymal stem cells. Ann Med 37: 469-479, 2005.

5. Rao MS and Mattson MP: Stem cells and aging: expanding the possibilities. Mech Ageing Dev 122: 713-734, 2001.

6. Zuk PA, Zhu M, Mizuno H, et al: Multilineage cells from human adipose tissue: implications for cell-based therapies. Tissue Eng 7: 211-228, 2001.

7. Sarugaser R, Lickorish D, Baksh D, et al: Human umbilical cord perivascular (HUCPV) cells: a source of mesenchymal progenitors. Stem Cells 23: 220-229, 2005.

8. Parolini O, Alviano F, Bagnara GP, et al: Isolation and characterization of cells from human term placenta: outcome of the first international workshop on placenta derived stem cells. Stem Cells 26: 300-311, 2008.

9. Soncini M, Vertua E, Gibelli L, et al: Isolation and characterization of mesenchymal cells from human fetal membranes. J Tissue Eng Regen Med 1: 296-305, 2007.
10. Bieback K, Kern S, Klüter H, et al: Critical parameters for the isolation of mesenchymal stem cells from umbilical cord blood. Stem Cells 22: 625-634, 2004.

11. Lee OK, Kuo TK, Chen WM, et al: Isolation of multipotent mesenchymal stem cells from umbilical cord blood. Blood 103: 1669-1675, 2004.

12. De CP, Bartsch GJ, Siddiqui MM, et al: Isolation of amniotic stem cell lines with potential for therapy. Nat Biotechnol 25: 100-106, 2007.

13. Lee OK, Kuo TK, Chen WM, et al: Isolation of multipotent mesenchymal stem cells from umbilical cord blood. Blood 103: 1669-1675, 2004.

14. Chan J, O'Donoghue K, Gavina M, et al: Galectin-1 induces skeletal muscle differentiation in human fetal mesenchymal stem cells and increases muscle regeneration. Stem Cells 24: 1879-1891, 2006.

15. Campagnoli C, Roberts IA, Kumar S, et al: Identification of mesenchymal stem/progenitor cells in human first-trimester fetal blood, liver, and bone marrow. Blood 98: 2396-2402, 2001.

16. Chan J, O'Donoghue K, de la Fuente J, et al: Human fetal mesenchymal stem cells as vehicles for gene delivery. Stem Cells 23: 93-102, 2005.

17. Chan J, Waddington SN, O'Donoghue K, et al: Widespread distribution and muscle differentiation of human fetal mesenchymal stem cells after intrauterine transplantation in dystrophic mdx mouse. Stem Cells 25: 875-884, 2007.

18. Miao ZN, Jin J, Chen L, et al: Isolation of mesenchymal stem cells from human placenta: comparison with human bone marrow mesenchymal stem cells. Cell Biol Int 30: 681-687, 2006.

19. Silva GV, Litovsky S, Assad JA, et al: Mesenchymal stem cells differentiate into an endothelial phenotype, enhance vascular density, and improve heart function in a canine chronic ischemia model. Circulation 111: 150-156, 2005.

20. Deng J, Petersen EB, Steindler DA, et al: Mesenchymal stem cells spontaneously express neural proteins in culture and are neurogenic after transplantation. Stem Cells 24: 1054-1064, 2006.

21. Li Y, Chopp M, Chen J, et al: Intrastriatal transplantation of bone marrow nonhematopoietic cells improves functional recovery after stroke in adult mice. J Cereb Blood Flow Metab 20: 1311-1319, 2000

22. Fukunaga A, Uchida K, Hara K, et al: Differentiation and angiogenesis of central nervous system stem cells implanted with mesenchyme into ischemic rat brain. Cell Transplant 8: 435-441, 1999.

23. Jori FP, Napolitano MA, Melone MA, et al: Molecular pathways involved in neural in vitro differentiation of marrow stromal stem cells. J Cellular Biochem 94: 645-655, 2005.

24. McCully JD, Wakiyama H, Hsieh YJ, et al: Differential contribution of necrosis and apoptosis in myocardial ischemiareperfusion injury. Am J Physiol Heart Circ Physiol 286: 1923-1935, 2004. 